\title{
THE STRUCTURE OF THE AUTOMORPHISM GROUP OF A FREE GROUP ON TWO GENERATORS
}

\author{
DRAGOMIR Ž. ĐOKOVIC
}

\begin{abstract}
Let $F_{2}=Z * Z$ be a free group of rank two. We show that Aut $F_{2}$ can be built up from cyclic groups by using only the free products and semidirect products. Explicitly we have Aut $F_{2}=\left((Z * Z) \rtimes\left(Z_{3} * Z_{3}\right)\right) \rtimes\left(Z_{4} \rtimes Z_{2}\right)$. As a corollary we obtain a simple presentation of Aut $F_{2}$.
\end{abstract}

Let $F_{2}=Z * Z=\langle x, y\rangle$ be a free group of rank 2 with generators $x$ and $y$, $Z_{3} * Z_{3}=\left\langle a, b: a^{3}=b^{3}=1\right\rangle$ the free product of two cyclic groups of order 3, and $D_{4}=Z_{4} \rtimes Z_{2}=\left\langle c, d: c^{4}=d^{2}=(c d)^{2}=1\right\rangle$ the dihedral group of order 8. (We use $*$ (resp. $\rtimes)$ to denote the free (resp. semidirect) product of two groups.) Let $\alpha, \beta \in$ Aut $F_{2}=\Phi$ be defined by

$$
\begin{aligned}
& \alpha: x \rightarrow x^{-1} y^{-1}, \quad y \rightarrow x ; \\
& \beta: x \rightarrow y^{-1}, \quad y \rightarrow x y^{-1} .
\end{aligned}
$$

Since $\alpha^{3}=\beta^{3}=1$ we have a homomorphism $Z_{3} * Z_{3} \rightarrow \Phi$ sending $a \rightarrow \alpha$ and $b \rightarrow \beta$. Let $H=F_{2} \rtimes\left(Z_{3} * Z_{3}\right)$ be the corresponding semidirect product. Thus $H$ is generated by $x, y, a, b$ with defining relations

$$
\begin{gathered}
a^{3}=b^{3}=1, \quad a x a^{-1}=x^{-1} y^{-1}, \quad a y a^{-1}=x, \\
b x b^{-1}=y^{-1}, \quad b y b^{-1}=x y^{-1} .
\end{gathered}
$$

Using (3) it is easy to verify that $H$ has endomorphisms $\gamma$ and $\delta$ such that

$$
\begin{aligned}
\gamma: x \rightarrow y^{-1}, \quad y \rightarrow x, \quad a \rightarrow b, & b \rightarrow x a ; \\
\delta: x \rightarrow y, \quad y \rightarrow x, \quad a \rightarrow y^{-1} a^{-1}, \quad b & \rightarrow b^{-1} .
\end{aligned}
$$

From (4) and (5) one derives that $\gamma^{4}=\delta^{2}=(\gamma \delta)^{2}=1$, and so there is a homomorphism $D_{4} \rightarrow$ Aut $H$ sending $c \rightarrow \gamma$ and $d \rightarrow \delta$. We let

$$
G=H \rtimes D_{4}=\left(F_{2} \rtimes\left(Z_{3} * Z_{3}\right)\right) \rtimes D_{4}
$$

be the corresponding semidirect product. Thus $G$ is generated by $x, y, a, b, c, d$ with defining relations those of $\mathrm{H}$ and $\mathrm{D}_{4}$ together with

$$
\begin{gathered}
c x c^{-1}=y^{-1}, \quad c y c^{-1}=x, \quad c a c^{-1}=b, \quad c b c^{-1}=x a, \\
d x d=y, \quad d a d=y^{-1} a^{-1}, \quad d b d=b^{-1} .
\end{gathered}
$$

Received by the editors April 22, 1982.

1980 Mathematics Subject Classification. Primary 20E05, 20F28, 20F05; Secondary 20E06.

Key words and phrases. Free product, semidirect product, automorphisms, finite presentation.

'Research supported by NSERC of Canada Grant A-5285. 
Clearly $F_{2} \triangleleft G$ and let $\phi: G \rightarrow \Phi$ be the canonical homomorphism: $\phi(z)$ is the restriction to $F_{2}$ of the inner automorphism $t \rightarrow z t z^{-1}$ of $G$.

THEOREM. $\phi$ is an isomorphism.

Proof. We have $F_{2} \triangleleft G$ and $F_{2} \triangleleft \Phi$ and the restriction of $\phi$ to $F_{2}$ is the identity map. Hence it suffices to show that the induced map $\bar{\phi}: \bar{G} \rightarrow \bar{\Phi}\left(\bar{G}=G / F_{2}\right.$ and $\left.\bar{\Phi}=\Phi / F_{2}\right)$ is an isomorphism. Let $\bar{F}_{2}=F_{2} / F_{2}^{\prime}$ where $F_{2}^{\prime}$ is the commutator subgroup of $F_{2}$. The group $\bar{F}_{2}$ is free abelian of rank 2 with a basis $\{\bar{x}, \bar{y}\}$. One knows [2, Proposition 4.5, p. 25] that the canonical homomorphism $\psi: \bar{\Phi} \rightarrow$ Aut $\bar{F}_{2}$ is an isomorphism. By using $\psi$ and the basis $\{\bar{x}, \bar{y}\}$ of $\bar{F}_{2}$ we shall identify $\bar{\Phi}$ with $\mathrm{GL}_{2}(Z)$. From (3) and (6) we deduce that

$$
\bar{\phi}(\bar{b})=\left(\begin{array}{rr}
0 & 1 \\
-1 & -1
\end{array}\right), \quad \bar{\phi}(\bar{c})=\left(\begin{array}{rr}
0 & 1 \\
-1 & 0
\end{array}\right), \quad \bar{\phi}(\bar{d})=\left(\begin{array}{ll}
0 & 1 \\
1 & 0
\end{array}\right) .
$$

Now we need the fact that $\mathrm{GL}_{2}(Z)$ is generated by

$$
A=\left(\begin{array}{rr}
0 & -1 \\
1 & 1
\end{array}\right), \quad B=\left(\begin{array}{rr}
0 & -1 \\
1 & 0
\end{array}\right), \quad C=\left(\begin{array}{ll}
0 & 1 \\
1 & 0
\end{array}\right)
$$

with defining relations (see [2, p. 25] or [1, Chapter 7])

$$
A^{6}=(A C)^{2}=(B C)^{2}=A^{3} B^{2}=1 .
$$

It follows from the definition of $G$ and $\bar{G}$ that

$$
\bar{b}^{3}=\bar{c}^{4}=(\bar{b} \bar{d})^{2}=\left(\bar{b} \bar{c}^{2}\right)^{2}=1 .
$$

Using (8) and (9) one can easily check that there is a homomorphism $\theta: \mathrm{GL}_{2}(Z) \rightarrow \bar{G}$ such that

$$
\theta: A \rightarrow \bar{b} \bar{c}^{2} . \quad B \rightarrow \bar{c}^{-1} . \quad C \rightarrow \bar{d} .
$$

Since $\bar{G}$ is generated by $\bar{b}, \bar{c}$ and $\bar{d}$ it follows from (7) and (10) that $\bar{\phi}$ and $\theta$ are inverses of each other.

As a corollary we can obtain a simple presentation of $\Phi$. For that purpose we shall first "simplify" the above presentation of $G$ by eliminating the redundant generators $x, y$ and $b$. Let $K$ be the group with generators $u, v, w$ and defining relations

$$
u^{3}=v^{4}=w^{2}=(v w)^{2}=v^{2} u v^{2} w u w=\left[u v u, v^{2}\right]=1 .
$$

LEMMA. There is an isomorphism $f: K \rightarrow G$ such that

$$
f: u \rightarrow a, \quad v \rightarrow c, \quad w \rightarrow d .
$$

Proof. In $G$ we have $a^{3}=c^{4}=d^{2}=(c d)^{2}=1, c^{2} a c^{2} d a d=\gamma^{2}(a) \delta(a)=$ $x a \cdot y^{-1} a^{-1}=x \alpha\left(y^{-1}\right)=1$, and

$$
\begin{aligned}
{\left[a c a, c^{2}\right] } & =a c a c^{2} a^{-1} c^{-1} a^{-1} c^{2}=a \gamma(a) \gamma^{-1}\left(a^{-1}\right) \gamma^{2}\left(a^{-1}\right) \\
& =a \cdot b \cdot b^{-1} y \cdot a^{-1} x^{-1}=\alpha(y) x^{-1}=1 .
\end{aligned}
$$

This proves that there exists a homomorphism $f: K \rightarrow G$ such that (12) holds. 
Similar routine (but longer) computations show that there is a homomorphism $g$ : $G \rightarrow K$ such that

$$
\begin{aligned}
x \rightarrow v^{2} u v^{2} u^{-1}, \quad y & \rightarrow\left(u^{-1} w\right)^{2}, \quad a \rightarrow u, \\
b & \rightarrow v u v^{-1}, \quad c \rightarrow v, \quad d \rightarrow w .
\end{aligned}
$$

From (12) and (13) it follows that $f$ and $g$ are inverses of each other.

Corollary. Let $\varepsilon$ (resp. $\zeta$ ) be the restriction of $\gamma$ (resp. $\delta$ ) to $F_{2}$. Then $\Phi$ is generated by $\alpha, \varepsilon, \zeta$ with defining relations

$$
\alpha^{3}=\varepsilon^{4}=\zeta^{2}=(\varepsilon \zeta)^{2}=\varepsilon^{2} \alpha \varepsilon^{2} \zeta \alpha \zeta=\left[\alpha \varepsilon \alpha, \varepsilon^{2}\right]=1 .
$$

Proof. This follows from the theorem, the lemma, and the fact that the isomorphism $\phi \circ f: K \rightarrow \Phi$ sends $u \rightarrow \alpha, v \rightarrow \varepsilon, w \rightarrow \zeta$.

In conclusion let us mention that another simple presentation of $\Phi$ is due to $\mathrm{B}$. $\mathrm{H}$. Neumann [4], see also [3, p. 169].

ACKNOWLEDGEMENT. I would like to thank Rutgers University for warm hospitality during a period when this paper was written.

\section{REFERENCES}

1. H. S. M. Coxeter and W. O. J. Moser, Generators and relations for discrete groups, Ergeb. Math. Grenzgeb., Bd. 14, 3rd ed., Springer-Verlag, Berlin, Heidelberg and New York, 1972.

2. R. C. Lyndon and P. E. Schupp, Combinatorial group theory, Ergeb. Math. Grenzgeb., Bd. 80, Springer-Verlag, Berlin, Heidelberg and New York, 1977.

3. W. Magnus, A. Karrass and D. Solitar, Combinatorial group theory, Wiley, New York, 1966.

4. B. H. Neumann, Die Automorphismengruppe der freien Gruppen, Math. Ann. 107 (1932), 367-386.

Department of Pure Mathematics, University of Waterloo, Waterloo, Ontario, N2L 3G1, CANADA 